\title{
AVIFAUNA OF THRISSUR DISTRICT, KERALA, INDIA
}

\author{
E.A. Jayson ${ }^{1}$ and C. Sivaperuman ${ }^{2}$
}

Division of Forest Ecology and Biodiversity Conservation, Kerala Forest Research Institute, Peechi, Thrissur, Kerala 680653, India Email: 1 jayson@kfri.org; ${ }^{2}$ C_sivaperuman@hotmail.com

\begin{abstract}
Three hundred and thirteen taxa of birds belonging to 64 Families were recorded in the Thrissur district, Kerala during a study on the avifauna there carried out from 1992 to 2002. 219 species of these were residents, 68 were transcontinental migrants, 26 were local migrants and one species was a straggler. The Order Passeriformes was highest in dominance followed by Charadriiformes, Ciconiiformes, Falconiformes, Coraciiformes and Piciformes. Seven species endemic to the Western Ghats and 11 species having threatened status were recorded. Out of the 1,340 species of birds recorded from the Indian subcontinent, 23\% were found in Thrissur district. This is the first attempt to compile district-wise distribution of avifauna in Kerala, which will pave way for a Bird Atlas of Kerala.
\end{abstract}

\section{KeYwORDS}

Avifauna, birds, checklist, Thrissur, Kerala, India

Scientific studies on the birds of Kerala state commenced with Hume $(1876,1878)$ reporting the first and second list of birds of Travancore. Later, many studies were conducted on forest species in the Western Ghats such as Ali (1969), Zacharias (1979), Gaston et al. (1979), Vijayan (1979), Shukkur and Joseph (1980), Yahya (1980), Vijayan (1984), Zacharias and Mathew (1988), Satheesan (1990), Neelakantan et al. (1993), Santharam (1995a), Joseph (1999), and Jayson and Mathew (2000a, 2000b, 2002, 2003). Several studies were reported from the Thrissur district also. Jairaj and Kumar (1990) recorded the Eurasian Spoonbill (Platalea leucoradia) from the Kole wetlands. Perennou (1990) reported four species of waterfowl from the same area. Nature Education Society Thrissur (NEST) reported the list of birds from Peechi-Vazhani Wildlife Sanctuary (Anon., 1991), and Kole wetlands, during the winter months of 1992 and 1993 (Anon., 1992, 1993). Santharam (1995b) recorded Plain Sand Martin (Riparia paludicola) from the Kole wetlands. Similarly, Jayson (2000) reported Black Stork (Ciconia nigra) from the same area. Sivaperuman and Jayson (2000) published the inventory of the birds of the Kole wetlands. Sivaperuman and Jayson (2002) recorded Northern Shoveller (Anas clypeata) from the Kole wetlands. Structure and species composition of birds of Kole wetlands were reported by Sivaperuman and Jayson (2001). The same authors reported the Lesser Frigate Bird Fregata ariel also from the Kole wetlands in 2003. Ravindran (2001) reported White-winged Black Tern from Thrissur. Even though many such records were available in the past, no effort was made to compile the avifauna of Thrissur District or any other districts in the state. An attempt is made here to compile the avifauna of Thrissur district based on the field surveys and published work.

\section{Study AREA}

Thrissur district located between $10^{\circ} 10^{\prime}-10^{\circ} 46^{\prime} \mathrm{N} \& 76^{0} 57^{\prime}-76^{0} 54^{\prime} \mathrm{E}$ is situated in the central region and is in the western slope of the southern Western Ghats (Figure 1). The extent of the area is $1,041 \mathrm{~km}^{2}$. The forests of Thrissur district fall within three administrative divisions of the Kerala Forest and Wildlife Department, viz. Thrissur, Chalakkudi and Vazhachal. Two wildlife sanctuaries, namely the Peechi-Vazhani and the Chimmony and a Ramsar site, the Vembanad-Kole wetlands exist in the district.

Topographically, the area is divisible into the Machad Mala Ridge, the Vellani Mala Ridge, the low-lying foothills of the Machad Mala Ridge, the Vellani Mala Ridge, the AnaikkalMangattukomban Ridge and the Sholayar and Parambikulam Valley. The highest point is Karimala Gopuram $(1,416 \mathrm{~m})$. The area is drained by several west flowing rivers namely Chalakkudi, Karappara, Sholayar, Canakkayam, Manali, Chimini Puzha, Mooply Puzha and Kurumali Puzha (Sasidharan \& Sivarajan, 1996).

The climate is moderate and there are three distinct seasons. The dry season (December to April), wet season-I (May to August) during the period of southwest monsoon and wet season-II (September to November) during northeast monsoon. The average daily maximum temperature during the hottest month is around $32^{\circ} \mathrm{C}$ in the coastal region and about $37^{\circ} \mathrm{C}$ in the interiors. The normal rainfall in the district is above $3000 \mathrm{~mm}$ and the relative humidity is normally over $70 \%$, especially during the monsoons.

The vegetation can be broadly divided into natural forest, plantations, agriculture fields, wetlands and coastal areas. Natural forests have been recognised into five forest types by Champion and Seth (1968). West-coast tropical evergreen forests are characterised by the profusion of species, particularly trees and woody climbers and the canopy is closed. The secondary species and exotic weeds are absent. The lower shrub layer is composed of mostly seedling and saplings of tree species. The evergreen forests are found between 400 $1,200 \mathrm{~m}$. It has been observed that there is difference in the physiognomy and composition of species with altitude. Westcoast semi-evergreen forests are found below $700 \mathrm{~m}$. It is intermediate between evergreen and moist deciduous type. Southern moist mixed deciduous forests are seen below $600 \mathrm{~m}$. Moist deciduous forests reveal their identity as the trees shed their leaves. The leafless period varies from a few weeks up to five months depending on the species. Southern montane wet temperate forests is characterised by the short-boled and highly branched trees. The branches are densely clothed with moss and other epiphytes. There is no stratification for trees and leaves in general are small. Southern montane wet grasslands 


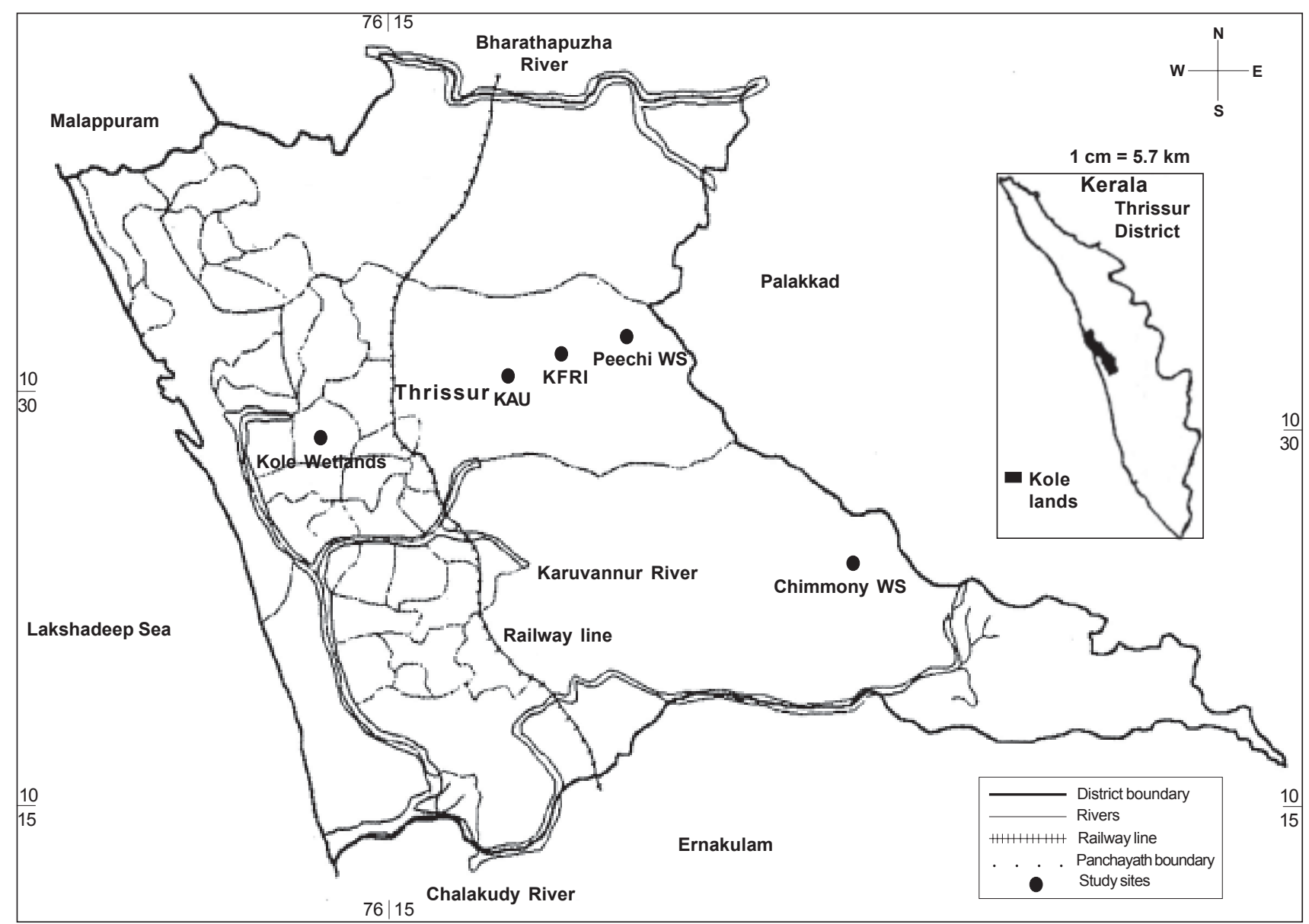

Figure 1. Thrissur District

are confined to the hilltop and grasses dominate the vegetation. About 20,000ha of area are under plantations with teak as the most extensively raised species in about 9,000 ha.

Kole wetland is an important wetland in the study area, which lies between $10^{\circ} 20^{\prime}-10^{\circ} 40^{\prime} \mathrm{N} \& 75^{\circ} 58^{\prime}-76^{\circ} 11^{\prime} \mathrm{E}$. The Kole wetland covers an area of 13,632ha, extending from the northern banks of Chalakudy river in the south to the southern banks of Bharathapuzha river in the north. A major portion of the area is flat and remains submerged for about six months in a year, during June to November. These lands were formerly shallow lagoons, which gradually were silted up. Two rivers mainly bring the floodwaters into the area, namely Kechery and Karuvannur, which finally empty into the Arabian Sea. The wetland is a low-lying tract, located $0.5 \mathrm{~m}$ to $1.0 \mathrm{~m}$ below mean sea level.

\section{Methods}

The study was mainly based on direct observational methods. Line transect method, Point count method and total count method were employed. Data was collected from five locations in the district representing forest, wetlands and agricultural fields. The sampling locations were Kole wetlands, Chimmony Wildlife Sanctuary, Peechi-Vazhani Wildlife Sanctuary, Kerala
Forest Research Institute (KFRI) campus, Kerala Agricultural University (KAU) campus and the data was collected between 1992 and 2002. Birds of Chimmony Wildlife Sanctuary were surveyed during the year 1992 to 1994, as a part of major ecological studies was included in the present study (Jayson \& Easa, 1996). Inventory of avifauna of Kole wetlands had been prepared as a part of detailed ecological studies during the year 1998 to 2002 viz. Jayson (2000), Sivaperuman and Jayson (2000), Jayson and Sivaperuman (2003). Birds of KFRI campus were recorded during 1995-2000 (Jayson et al., 2000). Bird census reports of Peechi-Vazhani Wildlife Sanctuary (Anon., 1991), Kole Wetlands (Anon., 1992, 1993) and KAU Campus (Nameer et al., 2000) were also consulted for compiling the inventory. Birds were classified as migratory or resident species based on Ali and Ripley (1983). The Common and scientific names are after Manakadan and Pittie (2001).

\section{RESULTS}

Three-hundred-and-thirteen taxa of birds were recorded from the Thrissur district, which belong to 66 families under 18 orders (Table 1). Out of these, 219 species were residents, 68 were trans-continental migrants, 26 were local migrants and one species was a straggler. The highest number of species was recorded from Kole wetlands (204), followed by Peechi-Vazhani 
Wildlife Sanctuary (202), Chimmoni Wildlife Sanctuary (168), KAU campus (131) and KFRI campus (94). The Order Passeriformes was highest in dominance followed by Charadriiformes, Ciconiiformes, Falconiformes, Coraciiformes and Piciformes (Fig. 2).

The highest number of birds recorded were: insectivores (117) followed by omnivores (66), aquatic feeders (45), carnivores (28), granivores (26), frugivores (19) and nectar-frugivores (12) (Table 2). Out of 16 species endemic to the Western Ghats, seven were recorded from the district, namely, Nilgiri Wood Pigeon, Blue-winged Parakeet, Malabar Grey Hornbill, Greyheaded Bulbul, Small Sunbird, Indian Rufous Babbler and White-bellied Blue Flycatcher. Out of the 315 species of birds found in the district, 11 species, namely, Spot-billed Pelican, Nilgiri Wood Pigeon, Darter, Painted Stork, Oriental White Ibis, Ferruginous Duck, Pallid Harrier, Greater Grey-headed Fisheagle, Red-headed Vulture, Great Pied-Hornbill and Wood Snipe were listed in the threatened birds of the world (BirdLife International, 2001).

A comparison of number of bird species recorded from Thrissur district with those from Kerala, and the world is given in Table 3. Out of the 1,340 species of birds recorded from the Indian subcontinent $23 \%$ cent are found in Thrissur district. When compared with the avifauna of Kerala $66 \%$ of the birds found in the state were recorded in the district.

\section{Discussion}

The high avian species richness recorded from the Thrissur district is due to the presence of diverse habitat types in the district. The high number of trans-continental migrants recorded in the district is associated with the presence of the Kole wetland in the district. The area supports the transcontinental migrants during the months of September to March. The avian species richness in the Peechi-Vazhani and Chimmoni wildlife sanctuaries is comparatively less, which is likely due to the low habitat diversity. Absence of wetlands here functioned as constrains for the wintering migratory species and only 19 species of wetland birds were recorded. On the other hand, passerine species dominated these two sites. KAU and KFRI campuses are located in the midst of human habitations and the extent of area is less due to which comparatively less number of birds were recorded from here. This compilation of distribution of the avifauna of Thrissur district is a first step towards the preparation of a Bird Atlas of Kerala.

\section{REFERENCES}

Ali, S. (1969). Birds of Kerala. Oxford University Press, 444pp.

Ali, S. and S.D. Ripley (1983). Hand Book of the Birds of India and Pakistan. Oxford University Press, Oxford, 737pp.

Anon. (1991). Birds of Peechi-Vazhani Wildlife Sanctuary: A survey report. Nature Education Society Thrissur (NEST), in collaboration with Kerala Forest Research Institute (KFRI) and Kerala Forest Department, $18 \mathrm{pp}$.

Anon. (1992). Birds of Kole wetlands: A survey report-I. Nature Education Society Thrissur (NEST), in collaboration with Kerala Forest Research Institute (KFRI) and Kerala Forest Department, 16pp.

Anon. (1993). Birds of Kole wetlands: A Survey Report-II. Nature
Table 2. Order and status of birds recorded from Thrissur District, Kerala

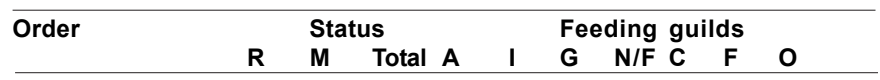

$\begin{array}{lllllllllll}\text { Podicipediformes } & 1 & - & 1 & 1 & - & - & - & - & - & - \\ \text { Pelecaniformes } & 5 & 2 & 7 & 7 & - & - & - & - & - & - \\ \text { Ciconiiformes } & 20 & 2 & 22 & 22 & - & - & - & - & - & - \\ \text { Anseriformes } & 4 & 6 & 10 & 10 & - & - & - & - & - & - \\ \text { Falconiformes } & 16 & 3 & 19 & - & - & - & - & 19 & - & - \\ \text { Galliformes } & 5 & - & 5 & - & 5 & 0 & - & - & - & - \\ \text { Gruiformes } & 7 & - & 7 & - & - & 7 & - & - & - & - \\ \text { Charadriiformes } & 9 & 49 & 58 & - & - & - & - & - & - & 58 \\ \text { Columbiformes } & 10 & - & 10 & - & - & 10 & - & - & - & - \\ \text { Psittaciformes } & 4 & - & 4 & - & - & - & - & - & 4 & - \\ \text { Cuculiformes } & 9 & - & 9 & - & - & - & - & - & 9 & - \\ \text { Strigiformes } & 9 & - & 9 & - & - & - & - & 9 & - & - \\ \text { Caprimulgiformes } & 5 & - & 5 & - & 5 & - & - & - & - & - \\ \text { Apodiformes } & 7 & - & 7 & - & 7 & - & - & - & - & - \\ \text { Trogoniformes } & 1 & - & 1 & - & 1 & - & - & - & - & - \\ \text { Coraciiformes } & 14 & - & 14 & 5 & 6 & - & - & - & - & 3 \\ \text { Piciformes } & 13 & - & 13 & - & 13 & - & - & - & - & 0 \\ \text { Passeriformes } & 105 & 7 & 112 & - & 80 & 09 & 12 & - & 6 & 5 \\ \text { Total } & \mathbf{2 4 4} & \mathbf{6 9} & \mathbf{3 1 3} & \mathbf{4 5} & \mathbf{1 1 7} & \mathbf{2 6} & \mathbf{1 2} & \mathbf{2 8} & \mathbf{1 9} & \mathbf{6 6} \\ \text { R } & & & \end{array}$

R - Resident; M - Migrants; A - Aquatic feeders; I - Insectivores;

C - Carnivores; G - Granivores; F - Frugivores; N/F - Nectar-frugivores; O - Omnivores

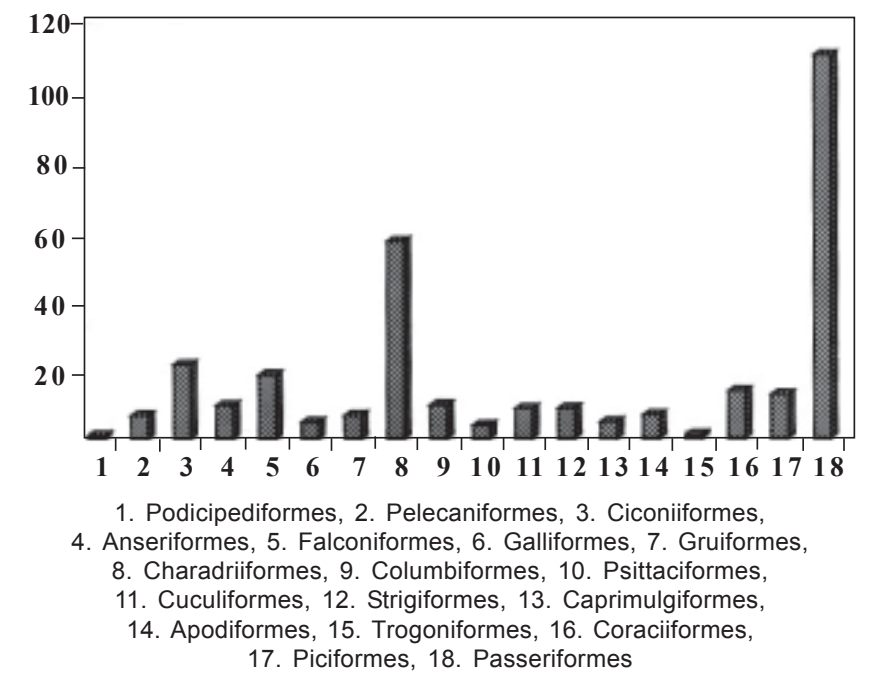

Figure. 2. Order wise distribution of birds in Thrissur District, Kerala

Education Society Thrissur (NEST), in collaboration with Kerala Forest Research Institute (KFRI) and Kerala Forest Department, 18pp.

BirdLife International (2001). Threatened Birds of Asia. The BirdLife International Red Data Book. Cambridge, UK, BirdLife International. Champion, H.G. and S.K. Seth (1968). A Revised Survey of the Forest Types of India. Govt. of India Press, Delhi.

Gaston, A.J., D.N. Mathew and V.J. Zacharias (1979). Regional variation in the breeding seasons of Babblers (Turdoides spp.) in India. Ibis 121(4): 512-516.

Daniels, R.J.R. (1998). A Field Guide to the Birds of Southwestern India. Oxford University Press, Delhi, 217pp.

Harrison, C.J.O. (1978). Bird Families of the World. Elsevier Phaidon, Oxford, $264 \mathrm{pp}$.

Hume, A.O. (1876). A first list of the birds of the Travancore hills. Stray Feathers 4(4,5\&6): 351-405.

Hume, A.O. (1878). A second list of the birds of southern Travancore. Stray Feathers 7(1-2): 33-39.

Jairaj, A.P. and V.K.S. Kumar (1990). Occurrence of Spoonbill 
Table 1. List of birds recorded from Thrissur district, Kerala

\begin{tabular}{|c|c|c|c|c|c|c|c|c|}
\hline & Common Name & Scientific Name & Status* & S1 & S2 & S3 & S4 & S5 \\
\hline \multicolumn{9}{|c|}{ Podicipedidae } \\
\hline 1. & Little Grebe & Tachybaptus ruficollis (Pallas) & $\mathrm{R}$ & $\sqrt{ }$ & & $\sqrt{ }$ & & \\
\hline \multicolumn{9}{|c|}{ Pelecanidae } \\
\hline 2. & Spot-billed Pelican & Pelecanus philippensis Gmelin & $\mathrm{R}$ & $\sqrt{ }$ & & & & \\
\hline \multicolumn{9}{|c|}{ Sulidae } \\
\hline 3. & Masked Booby & Sula dactylatra Lesson & M & $\sqrt{ }$ & & & & \\
\hline \multicolumn{9}{|c|}{ Phalacrocoracidae } \\
\hline 4. & Little Cormorant & Phalacrocorax niger (Vieillot) & $\mathrm{R}$ & $\sqrt{ }$ & & $\sqrt{ }$ & $\sqrt{ }$ & $\sqrt{ }$ \\
\hline 5. & Great Cormorant & Phalacrocorax carbo (Linnaeus) & LM & $\sqrt{ }$ & & & & \\
\hline 6. & Indian Shag & Phalacrocorax fuscicollis Stephens & LM & $\sqrt{ }$ & & & & \\
\hline \multicolumn{9}{|c|}{ Anhingidae } \\
\hline 7. & Darter & Anhinga melanogaster Pennant & LM & $\sqrt{ }$ & & $\sqrt{ }$ & $\sqrt{ }$ & $\sqrt{ }$ \\
\hline \multicolumn{9}{|c|}{ Fregatidae } \\
\hline 8. & Lesser Frigatebird & Fregata ariel (G.R. Gray) & $S$ & $\sqrt{ }$ & & & & \\
\hline \multicolumn{9}{|c|}{ Ardeidae } \\
\hline 9. & Grey Heron & Ardea cinerea Linnaeus & $\mathrm{R}$ & $\sqrt{ }$ & & & & \\
\hline 10. & Purple Heron & Ardea purpurea Linnaeus & $\mathrm{R}$ & $\sqrt{ }$ & & & & \\
\hline 11. & Little Green Heron & Butorides striatus (Linnaeus) & $\mathrm{R}$ & $\sqrt{ }$ & & $\sqrt{ }$ & $\sqrt{ }$ & $\sqrt{ }$ \\
\hline 12. & Indian Pond-Heron & Ardeola grayii Sykes & $\mathrm{R}$ & $\sqrt{ }$ & & $\sqrt{ }$ & $\sqrt{ }$ & $\sqrt{ }$ \\
\hline 13. & Cattle Egret & Bubulcus ibis Linnaeus & $\mathrm{R}$ & $\sqrt{ }$ & & $\sqrt{ }$ & $\sqrt{ }$ & $\sqrt{ }$ \\
\hline 14. & Large Egret & Casmerodius albus (Linnaeus) & $\mathrm{R}$ & $\sqrt{ }$ & & & & \\
\hline 15. & Median Egret & Mesophoyx intermedia (Wagler) & $\mathrm{R}$ & $\sqrt{ }$ & & & & \\
\hline 16. & Little Egret & Egretta garzetta (Linnaeus) & $\mathrm{R}$ & $\sqrt{ }$ & & $\sqrt{ }$ & $\sqrt{ }$ & $\sqrt{ }$ \\
\hline 17. & Western Reef-Heron & Egretta gularis (Bosc) & $\mathrm{R}$ & $\sqrt{ }$ & & & & \\
\hline 18. & Black-crowned Night- Heron & Nycticorax nycticorax Linnaeus & $\mathrm{R}$ & $\sqrt{ }$ & & $\sqrt{ }$ & $\sqrt{ }$ & $\sqrt{ }$ \\
\hline 19. & Chestnut Bittern & Ixobrychus cinnamomeus (Gmelin) & $\mathrm{R}$ & $\sqrt{ }$ & & $\sqrt{ }$ & & \\
\hline 20. & Yellow Bittern & Ixobrychus sinensis (Gmelin) & $\mathrm{R}$ & $\sqrt{ }$ & & & & \\
\hline 21. & Black Bittern & Dupetor flavicollis (Latham) & $\mathrm{R}$ & $\sqrt{ }$ & & $\sqrt{ }$ & $\sqrt{ }$ & $\sqrt{ }$ \\
\hline \multicolumn{9}{|c|}{ Ciconiidae } \\
\hline 22. & Painted Stork & Mycteria leucocephala (Pennant) & LM & $\sqrt{ }$ & & & & \\
\hline 23. & Asian Openbill-Stork & Anastomus oscitans Boddaert & LM & $\sqrt{ }$ & & & & \\
\hline 24. & Black Stork & Ciconia nigra (Linnaeus) & M & $\sqrt{ }$ & & & & \\
\hline 25. & White-necked Stork & Ciconia episcopus (Boddaert) & $\mathrm{R}$ & $\sqrt{ }$ & & & & \\
\hline 26. & European White Stork & Ciconia ciconia (Linnaeus) & M & $\sqrt{ }$ & & & & \\
\hline \multicolumn{9}{|c|}{ Threskiornithidae } \\
\hline 27. & Oriental White Ibis & Threskiornis melanocephalus (Latham) & LM & $\sqrt{ }$ & & & & \\
\hline 28. & Glossy Ibis & Plegadis falcinellus (Linnaeus) & LM & $\sqrt{ }$ & & & & \\
\hline 29. & Black Ibis & Pseudibis papillosa (Temminck) & LM & $\sqrt{ }$ & & & & \\
\hline 30. & Eurasian Spoonbill & Platalea leucorodia Linnaeus & LM & $\sqrt{ }$ & & & & \\
\hline \multicolumn{9}{|c|}{ Anatidae } \\
\hline 31. & Lesser Whistling-Duck & Dendrocygna javanica (Horsfield) & $\mathrm{R}$ & $\sqrt{ }$ & & $\sqrt{ }$ & & \\
\hline 32. & Northern Pintail & Anas acuta Linnaeus & M & $\sqrt{ }$ & & & & \\
\hline 33. & Ferruginous Pochard & Aythya nyroca (Guldenstadt) & M & $\sqrt{ }$ & & & & \\
\hline 34. & Common Teal & Anas crecca Linnaeus & M & $\sqrt{ }$ & & & & \\
\hline 35. & Spot-billed Duck & Anas poecilorhyncha J.R. Forester & LM & $\sqrt{ }$ & & & & \\
\hline 36. & Garganey & Anas querquedula Linnaeus & $\mathrm{M}$ & $\sqrt{ }$ & & & & \\
\hline 37. & Gadwall & Anas strepera Linnaeus & M & $\sqrt{ }$ & & & & \\
\hline 38. & Northern Shoveller & Anas clypeata Linnaeus & M & $\sqrt{ }$ & & & & \\
\hline 39. & Cotton Teal & Nettapus coromandelianus (Gmelin) & LM & $\sqrt{ }$ & & & & \\
\hline 40. & Comb Duck & Sarkidiornis melanotos (Pennant) & LM & $\sqrt{ }$ & & & & \\
\hline \multicolumn{9}{|c|}{ Accipitridae } \\
\hline 41. & Black-shouldered Kite & Elanus caeruleus (Desfontaines) & $\mathrm{R}$ & $\sqrt{ }$ & & $\sqrt{ }$ & $\sqrt{ }$ & $\sqrt{ }$ \\
\hline 42. & Black Kite & Milvus migrans (Boddaert) & $\mathrm{R}$ & $\sqrt{ }$ & $\sqrt{ }$ & $\sqrt{ }$ & $\sqrt{ }$ & $\sqrt{ }$ \\
\hline 43. & Brahminy Kite & Haliastur indus (Boddaert) & $\mathrm{R}$ & $\sqrt{ }$ & $\sqrt{ }$ & $\sqrt{ }$ & $\sqrt{ }$ & $\sqrt{ }$ \\
\hline 44. & Shikra & Accipiter badius (Gmelin) & $\mathrm{R}$ & $\sqrt{ }$ & $\sqrt{ }$ & $\sqrt{ }$ & $\sqrt{ }$ & $\sqrt{ }$ \\
\hline 45. & Eurasian Sparrow hawk & Accipiter nisus (Linnaeus) & M & $\sqrt{ }$ & $\sqrt{ }$ & & $\sqrt{ }$ & $\sqrt{ }$ \\
\hline 46. & Besra Sparrow hawk & Accipiter virgatus (Temminck) & $\mathrm{R}$ & & & & $\sqrt{ }$ & $\sqrt{ }$ \\
\hline 47. & Crested Goshawk & Accipiter trivirgatus (Temminck) & $\mathrm{R}$ & & & & $\sqrt{ }$ & $\sqrt{ }$ \\
\hline 48. & Changeable Hawk-Eagle & Spizaetus cirrhatus Gmelin & $\mathrm{R}$ & & & $\sqrt{ }$ & $\sqrt{ }$ & $\sqrt{ }$ \\
\hline 49. & Black Baza & Aviceda leuphotes (Dumont) & $\mathrm{R}$ & & & & $\sqrt{ }$ & $\sqrt{ }$ \\
\hline 50. & Oriental Honey-Buzzard & Pernis ptilorhynchus (Temminck) & $\mathrm{R}$ & & & & $\sqrt{ }$ & $\sqrt{ }$ \\
\hline 51. & Rufous-bellied Eagle & Hieraaetus kienerii (E.Geoffroy) & $\mathrm{R}$ & & & & $\sqrt{ }$ & $\sqrt{ }$ \\
\hline 52. & Black Eagle & Ictinaetus malayensis (Temminck) & $\mathrm{R}$ & & & & $\sqrt{ }$ & $\sqrt{ }$ \\
\hline 53. & Greater Grey-headed Fish Eagle & Icthyophaga ichthyaetus (Horsefield) & $\mathrm{R}$ & & & & $\sqrt{ }$ & $\sqrt{ }$ \\
\hline
\end{tabular}




\begin{tabular}{|c|c|c|c|c|c|c|c|c|}
\hline & Common Name & Scientific Name & Status* & S1 & S2 & S3 & S4 & S5 \\
\hline 54. & Red-headed Vulture & Sarcogyps calvus (Scopoli) & $\mathrm{R}$ & & & & $\sqrt{ }$ & $\sqrt{ }$ \\
\hline 55. & Pallid Harrier & Circus macrourus (S.G. Gmelin) & M & $\sqrt{ }$ & & & & \\
\hline 56. & Pied Harrier & Circus melanoleucos (Pennant) & M & $\sqrt{ }$ & & & & \\
\hline 57. & Western Marsh-Harrier & Circus aeruginosus (Linnaeus) & $\mathrm{R}$ & $\sqrt{ }$ & & & & \\
\hline 58. & Crested Serpent-Eagle & Spilornis cheela (Latham) & $\mathrm{R}$ & $\sqrt{ }$ & $\sqrt{ }$ & $\sqrt{ }$ & $\sqrt{ }$ & $\sqrt{ }$ \\
\hline \multicolumn{9}{|c|}{ Pandionidae } \\
\hline 59. & Osprey & Pandion haliaetus (Linnaeus) & $\mathrm{R}$ & $\sqrt{ }$ & & & $\sqrt{ }$ & $\sqrt{ }$ \\
\hline \multicolumn{9}{|c|}{ Phasianidae } \\
\hline 60. & Grey Francolin & Francolinus pondicerianus (Gmelin) & $\mathrm{R}$ & $\sqrt{ }$ & $\sqrt{ }$ & & $\sqrt{ }$ & $\sqrt{ }$ \\
\hline 61. & Jungle bush Quail & Perdicula asiatica (Latham) & $\mathrm{R}$ & & $\sqrt{ }$ & $\sqrt{ }$ & & $\sqrt{ }$ \\
\hline 62. & Red Spurfowl & Galloperdix spadicea (Gmelin) & $\mathrm{R}$ & $\sqrt{ }$ & $\sqrt{ }$ & $\sqrt{ }$ & $\sqrt{ }$ & $\sqrt{ }$ \\
\hline 63. & Grey Junglefowl & Gallus sonneratii Temminck & $\mathrm{R}$ & & $\sqrt{ }$ & $\sqrt{ }$ & & $\sqrt{ }$ \\
\hline 64. & Indian Peafowl & Pavo cristatus Linnaeus & $\mathrm{R}$ & $\sqrt{ }$ & $\sqrt{ }$ & & & \\
\hline \multicolumn{9}{|c|}{ Rallidae } \\
\hline 65. & Ruddy-breasted Crake & Porzana fusca (Linnaeus) & $\mathrm{R}$ & $\sqrt{ }$ & & $\sqrt{ }$ & & $\sqrt{ }$ \\
\hline 66. & Slaty-legged Crake & Rallina eurizonoides (Lafresnaye) & $\mathrm{R}$ & $\sqrt{ }$ & & & & $\sqrt{ }$ \\
\hline 67. & White-breasted Waterhen & Amaurornis phoenicurus (Pennant) & $\mathrm{R}$ & $\sqrt{ }$ & $\sqrt{ }$ & $\sqrt{ }$ & $\sqrt{ }$ & $\sqrt{ }$ \\
\hline 68. & Watercock & Gallicrex cinerea (Gmelin) & $\mathrm{R}$ & $\sqrt{ }$ & & & & $\sqrt{ }$ \\
\hline 69. & Common Moorhen & Gallinula chloropus (Linnaeus) & $\mathrm{R}$ & $\sqrt{ }$ & & & & \\
\hline 70. & Purple Moorhen & Porphyrio porphyrio (Linnaeus) & $\mathrm{R}$ & $\sqrt{ }$ & & & & \\
\hline 71. & Common Coot & Fulica atra Linnaeus & LM & $\sqrt{ }$ & & & & $\sqrt{ }$ \\
\hline \multicolumn{9}{|c|}{ Jacanidae } \\
\hline 72. & Pheasant-tailed Jacana & Hydrophasianus chirurgus (Scopoli) & LM & $\sqrt{ }$ & & & & \\
\hline 73. & Bronze-winged Jacana & Metopidius indicus (Latham) & $\mathrm{R}$ & $\sqrt{ }$ & & & & \\
\hline
\end{tabular}

\section{Rostratulidae}

74. Greater Painted-Snipe

Rostratula benghalensis (Linnaeus)

\section{$\mathrm{R}$}

Haematopodidae

75. Eurasian Oystercatcher

Haematopus ostralegus Linnaeus

M

Charadriidae

76. Red-wattled Lapwing

77. Yellow-wattled Lapwing

Pacific Golden-Plover

Grey Plover

Greater Sand Plover

Little Ringed Plover

Common Ringed Plover

Kentish Plover

Lesser Sand Plover

\section{Scolopacidae}

85. Whimbrel

86. Eurasian Curlew

87. Black-tailed Godwit

88. Bar-tailed Godwit

Common Redshank

Marsh Sandpiper

Common Greenshank

Green Sandpiper

Wood Sandpiper

Terek Sandpiper

Common Sandpiper

Ruddy Turnstone

Wood Snipe

Pintail Snipe

Common Snipe

Jack Snipe

Eurasian Woodcock

Great Knot

Little Stint

Temminck's Stint

Sanderling

Curlew Sandpiper

Dunlin

Broad-billed Sandpiper

Spoon-billed Sandpiper

110. Ruff

Vanellus indicus (Boddaert)

Vanellus malabaricus (Boddeart)

Pluvialis fulva (Gmelin)

Pluvialis squatarola (Linnaeus)

Charadrius leschenaultii Lesson

Charadrius dubius Scopoli

Charadrius hiaticula Linnaeus

Charadrius alexandrinus Linnaeus

Charadrius monogolus Pallas

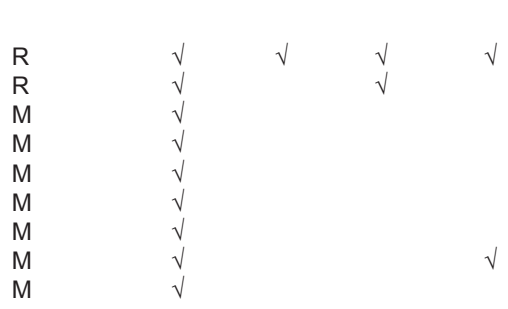

Numenius phaeopus (Linnaeus)

Numenius arquata Linnaeus

Limosa limosa (Linnaeus)

Limosa lapponica (Linnaeus)

Tringa totanus (Linnaeus)

Tringa stagnatilis (Bechstein)

Tringa nebularia (Gunner)

Tringa ochropus Linnaeus

Tringa glareola Linnaeus

Xenus cinereus (Guldenstadt)

Actitis hypoleucos Linnaeus

Arenaria interpres (Linnaeus)

Gallinago nemoricola Hodgson

Gallinago stenura (Bonaparte)

Gallinago gallinago (Linnaeus)

Lymnocryptes minimus (Brunnich)

Scolopax rusticola Linnaeus

Calidris tenuirostris (Horsfield)

Calidris minuta Leisler

Calidris temminckii (Leisler)

Calidris alba (Pallas)

Calidris ferruginea (Pontoppidan)

Calidris alpina (Linnaeus)

Limicola falcinellus (Pontoppidan)

Calidris pygmeus (Linnaeus)

Philomachus pugnax (Linnaeus)

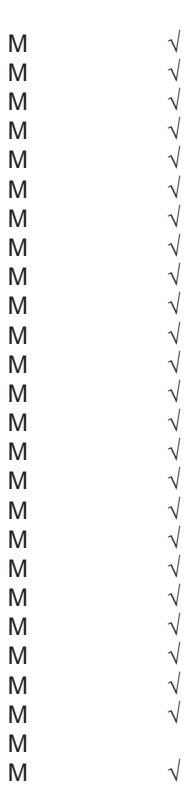

Recurvirostridae

111. __ Blackwinged Stilt

Himantopus himantopus (Linnaeus)

$\mathrm{M}$ 


\begin{tabular}{|c|c|c|c|c|c|c|c|c|}
\hline & Common Name & Scientific Name & Status* & s1 & S2 & S3 & S4 & S5 \\
\hline 112. & Pied Avocet & Recurvirostra avosetta Linnaeus & M & $\sqrt{ }$ & & & & \\
\hline \multicolumn{9}{|c|}{ Burhinidae } \\
\hline 113. & Stone-Curlew & Burhinus oedicnemus (Linnaeus) & LM & & & $\sqrt{ }$ & & \\
\hline 114. & Great Stone-Curlew & Esacus recurvirostris (Cuvier) & LM & & & & & $\sqrt{ }$ \\
\hline \multicolumn{9}{|c|}{ Glareolidae } \\
\hline 115. & Indian Courser & Cursorius coromandelicus (Gmelin) & M & & & & & \\
\hline 116. & Small Pratincole & Glareola lactea Temminck & LM & $\sqrt{ }$ & & & & $\sqrt{ }$ \\
\hline \multicolumn{9}{|c|}{ Laridae } \\
\hline 117. & Yellow-legged Gull & Larus cachinnans Pallas & M & $\sqrt{ }$ & & & & \\
\hline 118. & Lesser Black-backed Gull & Larus fuscus (Linnaeus) & M & $\sqrt{ }$ & & & & \\
\hline 119. & Brown-headed Gull & Larus brunnicephalus Jerdon & M & $\sqrt{ }$ & & & & \\
\hline 120. & Black-headed Gull & Larus ridibundus Linnaeus & M & $\sqrt{ }$ & & & & \\
\hline 121. & Whiskered Tern & Chlidonias hybridus (Pallas) & M & $\sqrt{ }$ & & $\sqrt{ }$ & & \\
\hline 122. & White-winged Black Tern & Chilidonias leucopterus & M & $\sqrt{ }$ & & & & \\
\hline 123. & Common Tern & Sterna hirundo Linnaeus & M & $\sqrt{ }$ & & & & $\sqrt{ }$ \\
\hline 124. & Gull-billed Tern & Gelochelidon nilotica (Gmelin) & M & $\sqrt{ }$ & & & & \\
\hline 125. & Caspian Tern & Sterna caspia Pallas & $\mathrm{M}$ & $\sqrt{ }$ & & & & \\
\hline 126. & River Tern & Sterna aurantia J.E.Gray & LM & $\sqrt{ }$ & & & & \\
\hline 127. & Black-bellied Tern & Sterna acuticauda J.E.Gray & $\mathrm{M}$ & $\sqrt{ }$ & & & & \\
\hline 128. & Little Tern & Sterna saundersi Hume & M & $\sqrt{ }$ & & & & \\
\hline 129. & Sandwich Tern & Sterna sandvicensis Latham & M & $\sqrt{ }$ & & & & \\
\hline \multicolumn{9}{|c|}{ Columbidae } \\
\hline 130. & Yellow-legged Green- Pigeon & Treron phoenicoptera (Latham) & $\mathrm{R}$ & & $\sqrt{ }$ & $\sqrt{ }$ & $\sqrt{ }$ & $\sqrt{ }$ \\
\hline 131. & Pompadour Green- Pigeon & Treron pompadora (Gmelin) & $\mathrm{R}$ & & & & $\sqrt{ }$ & $\sqrt{ }$ \\
\hline 132. & Green Imperial-Pigeon & Ducula aenea (Linnaeus) & $\mathrm{R}$ & & & & $\sqrt{ }$ & \\
\hline 133. & Mountain Imperial Pigeon & Ducula badia (Raffles) & $\mathrm{R}$ & & & & $\sqrt{ }$ & $\sqrt{ }$ \\
\hline 134. & Nilgiri Wood-Pigeon & Columba elphinstonii (Sykes) & $\mathrm{R}$ & & & & $\sqrt{ }$ & $\sqrt{ }$ \\
\hline 135. & Spotted Dove & Streptopelia chinensis (Scopoli) & $\mathrm{R}$ & $\sqrt{ }$ & $\sqrt{ }$ & $\sqrt{ }$ & & $\sqrt{ }$ \\
\hline 136. & Emerald Dove & Chalcophaps indica (Linnaeus) & $\mathrm{R}$ & & $\sqrt{ }$ & $\sqrt{ }$ & & \\
\hline 137. & Eurasian Collared Dove & Streptopelia decaocta (Frivaldszky) & $\mathrm{R}$ & $\sqrt{ }$ & & & $\sqrt{ }$ & \\
\hline 138. & Oriental Turtle-Dove & Streptopelia orientalis (Latham) & $\mathrm{R}$ & & & & $\sqrt{ }$ & $\sqrt{ }$ \\
\hline 139. & Blue Rock Pigeon & Columba livia Gmelin & $\mathrm{R}$ & $\sqrt{ }$ & $\sqrt{ }$ & $\sqrt{ }$ & $\sqrt{ }$ & $\sqrt{ }$ \\
\hline \multicolumn{9}{|c|}{ Psittacidae } \\
\hline 140. & Rose-ringed Parakeet & Psittacula krameri (Scopoli) & $\mathrm{R}$ & $\sqrt{ }$ & $\sqrt{ }$ & $\sqrt{ }$ & $\sqrt{ }$ & $\sqrt{ }$ \\
\hline 141. & Plum-headed Parakeet & Psittacula cyanocephala (Linnaeus) & $\mathrm{R}$ & $\sqrt{ }$ & $\sqrt{ }$ & $\sqrt{ }$ & $\sqrt{ }$ & $\sqrt{ }$ \\
\hline 142. & Blue-winged Parakeet & Psittacula columboides (Vigors) & $\mathrm{R}$ & & $\sqrt{ }$ & & $\sqrt{ }$ & $\sqrt{ }$ \\
\hline 143. & Indian Hanging Parrot & Loriculus vernalis (Sparrman) & $\mathrm{R}$ & & $\sqrt{ }$ & $\sqrt{ }$ & $\sqrt{ }$ & $\sqrt{ }$ \\
\hline \multicolumn{9}{|c|}{ Cuculidae } \\
\hline 144. & Pied Crested Cuckoo & Clamator jacobinus (Boddeart) & $\mathrm{R}$ & $\sqrt{ }$ & $\sqrt{ }$ & $\sqrt{ }$ & $\sqrt{ }$ & $\sqrt{ }$ \\
\hline 145. & Brainfever Bird & Hierococcyx varius Vahl & $\mathrm{R}$ & $\sqrt{ }$ & $\sqrt{ }$ & $\sqrt{ }$ & $\sqrt{ }$ & $\sqrt{ }$ \\
\hline 146. & Indian Cuckoo & Cuculus micropterus Gould & $\mathrm{R}$ & $\sqrt{ }$ & $\sqrt{ }$ & $\sqrt{ }$ & $\sqrt{ }$ & $\sqrt{ }$ \\
\hline 147. & Banded Bay Cuckoo & Cacomantis sonneratii Latham & $\mathrm{R}$ & $\sqrt{ }$ & $\sqrt{ }$ & $\sqrt{ }$ & $\sqrt{ }$ & $\sqrt{ }$ \\
\hline 148. & Indian Plaintive Cuckoo & Cacomantis passerinus (Vahl) & $\mathrm{R}$ & & & $\sqrt{ }$ & $\sqrt{ }$ & $\sqrt{ }$ \\
\hline 149. & Asian Koel & Eudynamys scolopacea (Linnaeus) & $\mathrm{R}$ & $\sqrt{ }$ & $\sqrt{ }$ & $\sqrt{ }$ & $\sqrt{ }$ & $\sqrt{ }$ \\
\hline 150. & Small Green-billed Malkoha & Phaenicophaeus viridirostris (Jerdon) & $\mathrm{R}$ & & & $\sqrt{ }$ & $\sqrt{ }$ & $\sqrt{ }$ \\
\hline 151. & Greater Coucal & Centropus sinensis (Stephens) & $\mathrm{R}$ & $\sqrt{ }$ & $\sqrt{ }$ & $\sqrt{ }$ & $\sqrt{ }$ & $\sqrt{ }$ \\
\hline 152. & Lesser Coucal & Centropus bengalensis (Gmelin) & $\mathrm{R}$ & & & $\sqrt{ }$ & & $\sqrt{ }$ \\
\hline \multicolumn{9}{|c|}{ Tytonidae } \\
\hline 153. & Barn Owl & Tyto alba (Scopoli) & $\mathrm{R}$ & $\sqrt{ }$ & $\sqrt{ }$ & $\sqrt{ }$ & $\sqrt{ }$ & $\sqrt{ }$ \\
\hline \multicolumn{9}{|c|}{ Strigidae } \\
\hline 154. & Collared Scops Owl & Otus bakkamoena Pennant & $\mathrm{R}$ & & & $\sqrt{ }$ & $\sqrt{ }$ & $\sqrt{ }$ \\
\hline 155. & Eurasian Scops-Owl & Otus scops (Linnaeus) & $\mathrm{R}$ & & & & $\sqrt{ }$ & $\sqrt{ }$ \\
\hline 156. & Brown Fish-Owl & Ketupa zeylonensis (Gmelin) & $\mathrm{R}$ & & & $\sqrt{ }$ & $\sqrt{ }$ & $\sqrt{ }$ \\
\hline 157. & Jungle Owlet & Glaucidium radiatum (Tickell) & $\mathrm{R}$ & & & $\sqrt{ }$ & $\sqrt{ }$ & $\sqrt{ }$ \\
\hline 158. & Spotted Owlet & Athene brama (Temminck) & $\mathrm{R}$ & $\sqrt{ }$ & $\sqrt{ }$ & $\sqrt{ }$ & & \\
\hline 159. & Brown Hawk-Owl & Ninox scutulata (Raffles) & $\mathrm{R}$ & & & $\sqrt{ }$ & $\sqrt{ }$ & $\sqrt{ }$ \\
\hline 160. & Mottled Wood-Owl & Strix ocellata (Lesson) & $\mathrm{R}$ & $\sqrt{ }$ & & $\sqrt{ }$ & & \\
\hline 161. & Brown Wood-Owl & Strix leptogrammica Temminck & $\mathrm{R}$ & & & & $\sqrt{ }$ & $\sqrt{ }$ \\
\hline \multicolumn{9}{|c|}{ Podargidae } \\
\hline 162. & Ceylon Frogmouth & Batrachostomus moniliger Blyth & $\mathrm{R}$ & & & & $\sqrt{ }$ & $\sqrt{ }$ \\
\hline \multicolumn{9}{|c|}{ Caprimulgidae } \\
\hline 163. & Great Eared Nightjar & Eurostopodus macrotis (Vigors) & $\mathrm{R}$ & & & & & $\sqrt{ }$ \\
\hline 164. & Indian Jungle Nightjar & Caprimulgus indicus Latham & $\mathrm{R}$ & & $\sqrt{ }$ & $\sqrt{ }$ & $\sqrt{ }$ & $\sqrt{ }$ \\
\hline 165. & Franklin's Nightjar & Caprimulgus affinis Horsfield & $\mathrm{R}$ & & & $\sqrt{ }$ & & $\sqrt{ }$ \\
\hline 166. & Common Indian Nightjar & Caprimulgus asiaticus Latham & $\mathrm{R}$ & & $\sqrt{ }$ & $\sqrt{ }$ & & $\sqrt{ }$ \\
\hline
\end{tabular}




\begin{tabular}{|c|c|c|c|c|c|c|c|c|}
\hline & Common Name & Scientific Name & Status* & s1 & S2 & S3 & S4 & S5 \\
\hline \multicolumn{9}{|c|}{ Apodidae } \\
\hline 167. & Indian Edible-nest Swiftlet & Collocalia unicolor (Jerdon) & $\mathrm{R}$ & & & & $\sqrt{ }$ & $\sqrt{ }$ \\
\hline 168. & Brown-backed Needle-tail Swift & Hirundapus giganteus (Temminck) & $\mathrm{R}$ & & & & $\sqrt{ }$ & $\sqrt{ }$ \\
\hline 169. & Whiterumped Spinetail Swift & Zoonavena sylvatica (Tickell) & $\mathrm{R}$ & & & & $\sqrt{ }$ & $\sqrt{ }$ \\
\hline 170. & Alpine Swift & Tachymarptis melba (Linnaeus) & $\mathrm{R}$ & $\sqrt{ }$ & $\sqrt{ }$ & & & $\sqrt{ }$ \\
\hline 171. & House Swift & Apus affinis (J.E. Gray) & $\mathrm{R}$ & $\sqrt{ }$ & $\sqrt{ }$ & $\sqrt{ }$ & & \\
\hline 172. & Asian Palm Swift & Cypsiurus balasiensis (J.E. Gray) & $\mathrm{R}$ & $\sqrt{ }$ & $\sqrt{ }$ & $\sqrt{ }$ & $\sqrt{ }$ & $\sqrt{ }$ \\
\hline \multicolumn{9}{|c|}{ Hemiprocnidae } \\
\hline 173. & Crested Tree-Swift & Hemiprocne coronata (Tickell) & $\mathrm{R}$ & & & $\sqrt{ }$ & $\sqrt{ }$ & $\sqrt{ }$ \\
\hline \multicolumn{9}{|c|}{ Trogonidae } \\
\hline 174. & Malabar Trogon & Harpactes fasciatus (Pennant) & $\mathrm{R}$ & & & & $\sqrt{ }$ & $\sqrt{ }$ \\
\hline \multicolumn{9}{|c|}{ Alcedinidae } \\
\hline 175. & Lesser Pied Kingfisher & Ceryle rudis (Linnaeus) & $\mathrm{R}$ & $\sqrt{ }$ & $\sqrt{ }$ & $\sqrt{ }$ & $\sqrt{ }$ & $\sqrt{ }$ \\
\hline 176. & Small Blue Kingfisher & Alcedo atthis (Linnaeus) & $\mathrm{R}$ & $\sqrt{ }$ & $\sqrt{ }$ & $\sqrt{ }$ & $\sqrt{ }$ & $\sqrt{ }$ \\
\hline 177. & Stork-billed Kingfisher & Halcyon capensis (Linnaeus) & $\mathrm{R}$ & $\sqrt{ }$ & $\sqrt{ }$ & $\sqrt{ }$ & $\sqrt{ }$ & $\sqrt{ }$ \\
\hline 178. & White-breasted Kingfisher & Halcyon smyrnensis (Linnaeus) & $\mathrm{R}$ & $\sqrt{ }$ & $\sqrt{ }$ & $\sqrt{ }$ & $\sqrt{ }$ & $\sqrt{ }$ \\
\hline 179. & Black-capped Kingfisher & Halcyon pileata (Boddaert) & $\mathrm{R}$ & $\sqrt{ }$ & & & & \\
\hline \multicolumn{9}{|c|}{ Meropidae } \\
\hline 180. & Chestnut-headed Bee-eater & Merops leschenaulti Vieillot & $\mathrm{R}$ & & $\sqrt{ }$ & $\sqrt{ }$ & $\sqrt{ }$ & \\
\hline 181. & Blue-tailed Bee-eater & Merops philippinus Linnaeus & LM & $\sqrt{ }$ & $\sqrt{ }$ & $\sqrt{ }$ & & $\sqrt{ }$ \\
\hline 182. & Small Bee-eater & Merops orientalis Latham & $\mathrm{R}$ & $\sqrt{ }$ & $\sqrt{ }$ & $\sqrt{ }$ & $\sqrt{ }$ & $\sqrt{ }$ \\
\hline 183. & Blue-bearded Bee-eater & Nyctyornis athertoni (Jardine \& Selby) & $\mathrm{R}$ & & & $\sqrt{ }$ & & \\
\hline \multicolumn{9}{|c|}{ Coraciidae } \\
\hline 184. & Indian Roller & Coracias benghalensis (Linnaeus) & $\mathrm{R}$ & $\sqrt{ }$ & $\sqrt{ }$ & $\sqrt{ }$ & $\sqrt{ }$ & $\sqrt{ }$ \\
\hline \multicolumn{9}{|c|}{ Upupidae } \\
\hline 185. & Common Hoopoe & Upupa epops Linnaeus & $\mathrm{R}$ & $\sqrt{ }$ & $\sqrt{ }$ & $\sqrt{ }$ & $\sqrt{ }$ & $\sqrt{ }$ \\
\hline \multicolumn{9}{|c|}{ Bucerotidae } \\
\hline 186. & Malabar Grey Hornbill & Ocyceros griseus (Latham) & $\mathrm{R}$ & & & & $\sqrt{ }$ & $\sqrt{ }$ \\
\hline 187. & Indian Grey Hornbill & Ocyceros birostris (Scopoli) & $\mathrm{R}$ & & $\sqrt{ }$ & $\sqrt{ }$ & & $\sqrt{ }$ \\
\hline 188. & Great Pied Hornbill & Buceros bicornis (Linnaeus) & $\mathrm{R}$ & & & & $\sqrt{ }$ & $\sqrt{ }$ \\
\hline \multicolumn{9}{|c|}{ Capitonidae } \\
\hline 189. & White-cheeked Barbet & Megalaima viridis (Boddaert) & $\mathrm{R}$ & $\sqrt{ }$ & $\sqrt{ }$ & $\sqrt{ }$ & $\sqrt{ }$ & $\sqrt{ }$ \\
\hline 190. & Crimson-throated Barbet & Megalaima rubricapilla (Gmelin) & $\mathrm{R}$ & & & & $\sqrt{ }$ & $\sqrt{ }$ \\
\hline 191. & Coppersmith Barbet & Megalaima haemacephala (P.L.S. Muller) & $\mathrm{R}$ & & $\sqrt{ }$ & $\sqrt{ }$ & $\sqrt{ }$ & \\
\hline \multicolumn{9}{|c|}{ Picidae } \\
\hline 192. & Rufous Woodpecker & Celeus brachyurus (Vieillot) & $\mathrm{R}$ & & $\sqrt{ }$ & $\sqrt{ }$ & $\sqrt{ }$ & $\sqrt{ }$ \\
\hline 193. & Small Yellow-naped Woodpecker & Picus chlorolophus Vieillot & $\mathrm{R}$ & & & $\sqrt{ }$ & $\sqrt{ }$ & \\
\hline 194. & $\begin{array}{l}\text { Little Scaly-bellied } \\
\text { Green Woodpecker }\end{array}$ & $\begin{array}{l}\text { Picus xanthopygaeus } \\
\text { (J.E. Grat \& G.R. Gray) }\end{array}$ & $\mathrm{R}$ & & & $\sqrt{ }$ & $\sqrt{ }$ & \\
\hline 195. & $\begin{array}{l}\text { Lesser Golden-backed } \\
\text { Woodpecker }\end{array}$ & Dinopium benghalense (Linnaeus) & $\mathrm{R}$ & $\sqrt{ }$ & $\sqrt{ }$ & $\sqrt{ }$ & $\sqrt{ }$ & $\sqrt{ }$ \\
\hline 196. & $\begin{array}{l}\text { Common Golden-backed } \\
\text { Woodpecker }\end{array}$ & Dinopium javanense (Ljungh) & $\mathrm{R}$ & & & & $\sqrt{ }$ & $\sqrt{ }$ \\
\hline 197. & Great Black Woodpecker & Dryocopus javensis (Horsfield) & $\mathrm{R}$ & & & & $\sqrt{ }$ & $\sqrt{ }$ \\
\hline 198. & Yellow-fronted Woodpecker & Dendrocopos mahrattensis (Latham) & $\mathrm{R}$ & & $\sqrt{ }$ & $\sqrt{ }$ & $\sqrt{ }$ & $\sqrt{ }$ \\
\hline 199. & Brown-capped Pygmy Woodpecke & er Dendrocopos nanus (Vigors) & $\mathrm{R}$ & & $\sqrt{ }$ & $\sqrt{ }$ & $\sqrt{ }$ & $\sqrt{ }$ \\
\hline 200. & Heart-spotted Woodpecker & Hemicircus canente (Lesson) & $\mathrm{R}$ & & $\sqrt{ }$ & & $\sqrt{ }$ & $\sqrt{ }$ \\
\hline 201. & Greater Golden-backed Woodpec & cker Chrysocolaptes lucidus (Scopoli) & $\mathrm{R}$ & & & $\sqrt{ }$ & $\sqrt{ }$ & $\sqrt{ }$ \\
\hline \multicolumn{9}{|c|}{ Pittidae } \\
\hline 202. & Indian Pitta & Pitta brachyura Linnaeus & M & & $\sqrt{ }$ & & & $\sqrt{ }$ \\
\hline \multicolumn{9}{|c|}{ Alaudidae } \\
\hline 203. & Ashy-crowned Sparrow-Lark & Eremopterix grisea (Scopoli) & $\mathrm{R}$ & $\sqrt{ }$ & & $\sqrt{ }$ & & $\sqrt{ }$ \\
\hline 204. & Bengal Bush Lark & Mirafra assamica Horsfield & $\mathrm{R}$ & & $\sqrt{ }$ & $\sqrt{ }$ & & $\sqrt{ }$ \\
\hline 205. & Malabar Crested Lark & Galerida malabarica (Scopoli) & $\mathrm{R}$ & $\sqrt{ }$ & & & $\sqrt{ }$ & $\sqrt{ }$ \\
\hline 206. & Eastern Skylark & Alauda gulgula Franklin & $\mathrm{R}$ & $\sqrt{ }$ & & & & $\sqrt{ }$ \\
\hline \multicolumn{9}{|c|}{ Hirundinidae } \\
\hline 207. & Plain Martin & Riparia paludicola (Vieillot) & $\mathrm{R}$ & & & & & $\sqrt{ }$ \\
\hline 208. & House Swallow & Hirundo tahitica Gmelin & $\mathrm{R}$ & $\sqrt{ }$ & & & $\sqrt{ }$ & $\sqrt{ }$ \\
\hline 209. & Common Swallow & Hirundo rustica Linnaeus & $\mathrm{R}$ & $\sqrt{ }$ & $\sqrt{ }$ & & $\sqrt{ }$ & $\sqrt{ }$ \\
\hline 210. & Red-rumped Swallow & Hirundo daurica Linnaeus & LM & $\sqrt{ }$ & $\sqrt{ }$ & $\sqrt{ }$ & $\sqrt{ }$ & $\sqrt{ }$ \\
\hline \multicolumn{9}{|c|}{ Motacillidae } \\
\hline 211. & Paddyfield Pipit & Anthus rufulus Vieillot & $\mathrm{R}$ & $\sqrt{ }$ & & $\sqrt{ }$ & & $\sqrt{ }$ \\
\hline 212. & Tree Pipit & Anthus trivialis (Linnaeus) & $M$ & $\sqrt{ }$ & & & & $\sqrt{ }$ \\
\hline
\end{tabular}




\begin{tabular}{|c|c|c|c|c|c|c|c|c|}
\hline & Common Name & Scientific Name & Status* & S1 & S2 & S3 & S4 & S5 \\
\hline 213. & Forest Wagtail & Dendronanthus indicus (Gmelin) & $\mathrm{R}$ & & & $\sqrt{ }$ & $\sqrt{ }$ & $\sqrt{ }$ \\
\hline 214. & Yellow Wagtail & Motacilla flava Linnaeus & LM & $\sqrt{ }$ & $\sqrt{ }$ & & $\sqrt{ }$ & $\sqrt{ }$ \\
\hline 215. & Citrine Wagtail & Motacilla citreola (Pallas) & LM & $\sqrt{ }$ & & & & \\
\hline 216. & Grey Wagtail & Motacilla cinerea Tunstall & LM & $\sqrt{ }$ & & $\sqrt{ }$ & $\sqrt{ }$ & $\sqrt{ }$ \\
\hline 217. & White Wagtail & Motacilla alba Linnaeus & LM & & & & $\sqrt{ }$ & $\sqrt{ }$ \\
\hline 218. & Large Pied Wagtail & Motacilla maderaspatensis Gmelin & $\mathrm{R}$ & $\sqrt{ }$ & $\sqrt{ }$ & $\sqrt{ }$ & $\sqrt{ }$ & $\sqrt{ }$ \\
\hline
\end{tabular}

Campephagidae

219. Pied Flycatcher-Shrike

220. Large Woodshrike

221. Common Wood-Shrike

222. Large Cuckoo-Shrike

223. Black-headed Cuckoo-Shrike

224. Scarlet Minivet

225. Small Minivet

Hemipus picatus (Sykes)

Tephrodornis gularis (Raffles)

Tephrodornis pondicerianus Gmelin

Coracina macei (Lesson)

Coracina melanoptera (Ruppell)

Pericrocotus flammeus (Forster)

Pericrocotus cinnamomeus (Linnaeus)

\begin{tabular}{|c|c|c|c|}
\hline $\mathrm{R}$ & & & $\sqrt{ }$ \\
\hline $\mathrm{R}$ & & & $\sqrt{ }$ \\
\hline$R$ & & $\sqrt{ }$ & $\sqrt{ }$ \\
\hline $\mathrm{R}$ & & $\sqrt{ }$ & $\sqrt{ }$ \\
\hline $\mathrm{R}$ & & $\sqrt{ }$ & $\sqrt{ }$ \\
\hline $\mathrm{R}$ & $\sqrt{ }$ & $\sqrt{ }$ & $\sqrt{ }$ \\
\hline$R$ & $\sqrt{ }$ & $\sqrt{ }$ & $\sqrt{ }$ \\
\hline
\end{tabular}

Pycnonotidae

226. Grey-headed Bulbul

227. Black-crested Bulbul

Red-whiskered Bulbul

Red-vented Bulbul

Yellow-browed Bulbul

231. White-browed Bulbul

Irenidae

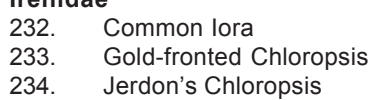

235. Asian Fairy-Bluebird

Lanidae

236. Brown Shrike

Pycnonotus priocephalus (Jerdon)

Pycnonotus melanicterus (Gmelin)

Pycnonotus jocosus (Linnaeus)

Pycnonotus cafer (Linnaeus)

lole indica (Jerdon)

Pycnonotus luteolus (Lesson)

Aegithina tiphia Linnaeus

Chloropsis aurifrons (Temminck)

Chloropsis cochinchinensis (Gmelin)

Irena puella (Latham)

\begin{tabular}{|c|c|c|c|c|}
\hline $\mathrm{R}$ & & & & $\sqrt{ }$ \\
\hline $\mathrm{R}$ & & & & $\sqrt{ }$ \\
\hline$R$ & $\sqrt{ }$ & $\sqrt{ }$ & $\sqrt{ }$ & $\sqrt{ }$ \\
\hline $\mathrm{R}$ & $\sqrt{ }$ & $\sqrt{ }$ & $\sqrt{ }$ & $\sqrt{ }$ \\
\hline $\mathrm{R}$ & & & & $\sqrt{ }$ \\
\hline $\mathrm{R}$ & & & $\sqrt{ }$ & $\sqrt{ }$ \\
\hline
\end{tabular}

Turdinae

237. Bluethroat

Oriental Magpie Robin

White-rumped Shama

Black Redstart

Pied Bushchat

Desert Wheatear

Indian Robin

Blue-headed Rock-Thrush

Malabar Whistling-Thrush

Orange-headed Thrush

247. Eurasian Blackbird

Timaliinae

248. Spotted Babbler

249. Black-headed Babbler

250. Common Babbler

251. Indian Rufous Babbler

Jungle Babbler

White-headed Babbler

254. Quaker Tit-Babbler

Sylviinae

255. Streaked Fantail-Warbler

256. Franklin's Prinia

257. Plain Prinia

258. Ashy Prinia

259. Common Tailorbird

260. Indian Great Reed- Warbler

261. Blyth's Reed-Warbler

262. Greenish Leaf-Warbler

263. Western Crowned Warbler

Lanius cristatus Linnaeus

Luscinia svecica (Linnaeus)

Copsychus saularis (Linnaeus)

Copsychus malabaricus (Scopoli)

Phoenicurus ochruros (Gmelin)

Saxicola caprata (Linnaeus)

Oenanthe deserti (Temminck)

Saxicoloides fulicata (Linnaeus)

Monticola cinclorhynchus (Vigors)

Myiophoneus horsfieldii (Vigors)

Zoothera citrina (Latham)

Turdus merula Lafresnay

$\mathrm{R}$

$R$

$\mathrm{R}$

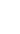

Pellorneum ruficeps Swainson Rhopocichla atriceps (Jerdon)

Turdoides caudatus (Dumont)

Turdoides subrufus (Jerdon)

Turdoides striatus Jerdon

Turdoides affinis (Jerdon)

Alcippe poioicephala (Jerdon)

Cisticola juncidis (Rafinesque)

Prinia hodgsonii Blyth

Prinia inornata Sykes

Prinia socialis Sykes

Orthotomus sutorius Pennant

Acrocephalus dumetorum Blyth \& Ehrenberg) $\mathrm{R}$

Phylloscopus trochiloides (Sundevall) M

Phylloscopus occipitalis (Blyth) M

\section{Muscicapinae}

264. Asian Brown Flycatcher

265. Brown-breasted Flycatcher

266. Rusty-tailed Flycatcher

267. Red-throated Flycatcher

268. White-bellied Blue Flycatcher

269. Blue-throated Flycatcher

270. Tickell's Blue Flycatcher

Muscicapa daurica (Pallas)

Muscicapa muttui (Layard)

Muscicapa ruficauda Swainson

Ficedula parva (Bechstein)

Cyornis pallipes (Jerdon)

Cyornis rubeculoides (Vigors)

Cyornis tickelliae Blyth

$\begin{array}{llllll}R & & & & & \sqrt{ } \\ R & \sqrt{ } & \sqrt{ } & \sqrt{ } & \sqrt{ } & \sqrt{ } \\ \mathrm{R} & & & & \sqrt{ } & \sqrt{ } \\ \mathrm{R} & \sqrt{ } & \sqrt{ } & & & \sqrt{ } \\ \mathrm{R} & \sqrt{ } & & & & \sqrt{ } \\ \mathrm{R} & \sqrt{ } & \sqrt{ } & \sqrt{ } & & \\ \mathrm{R} & & & & \sqrt{ } & \sqrt{ } \\ \mathrm{R} & & & & \sqrt{ } & \sqrt{ } \\ \mathrm{R} & & \sqrt{ } & \sqrt{ } & \sqrt{ } \\ \mathrm{R} & & \sqrt{ } & \sqrt{ } & \sqrt{ } \\ \mathrm{R} & & & \sqrt{ }\end{array}$




\begin{tabular}{|c|c|c|c|c|c|c|c|c|}
\hline & Common Name & Scientific Name & Status* & S1 & S2 & S3 & S4 & S5 \\
\hline \multicolumn{9}{|c|}{ Monarchinae } \\
\hline 271. & Asian Paradise Flycatcher & Terpsiphone paradisi (Linnaeus) & LM & $\sqrt{ }$ & $\sqrt{ }$ & $\sqrt{ }$ & $\sqrt{ }$ & $\sqrt{ }$ \\
\hline 272. & Black-naped Monarch-Flycatcher & Hypothymis azurea (Boddaert) & $\mathrm{R}$ & & & & $\sqrt{ }$ & $\sqrt{ }$ \\
\hline \multicolumn{9}{|c|}{ Rhipidurinae } \\
\hline 273. & White-browed Fantail-Flycatcher & Rhipidura aureola Lesson & $\mathrm{R}$ & & & $\sqrt{ }$ & & \\
\hline \multicolumn{9}{|c|}{ Paridae } \\
\hline 274. & Grey Tit & Parus major Linnaeus & $\mathrm{R}$ & & $\sqrt{ }$ & & $\sqrt{ }$ & $\sqrt{ }$ \\
\hline 275. & Black-lored Yellow-Tit & Parus xanthogenys Vigors & $\mathrm{R}$ & & & & $\sqrt{ }$ & $\sqrt{ }$ \\
\hline \multicolumn{9}{|c|}{ Sittidae } \\
\hline 276. & Velvetfronted Nuthatch & Sitta frontalis Swainson & $\mathrm{R}$ & & & & $\sqrt{ }$ & $\sqrt{ }$ \\
\hline \multicolumn{9}{|c|}{ Dicaeidae } \\
\hline 277. & Thick-billed Flowerpecker & Dicaeum agile (Tickell) & $\mathrm{R}$ & $\sqrt{ }$ & $\sqrt{ }$ & & $\sqrt{ }$ & $\sqrt{ }$ \\
\hline 278. & Tickell's Flowerpecker & Dicaeum erythrorhynchos (Latham) & $\mathrm{R}$ & $\sqrt{ }$ & $\sqrt{ }$ & $\sqrt{ }$ & $\sqrt{ }$ & $\sqrt{ }$ \\
\hline 279. & Plain Flowerpecker & Dicaeum concolor Jerdon & $\mathrm{R}$ & & & $\sqrt{ }$ & $\sqrt{ }$ & $\sqrt{ }$ \\
\hline \multicolumn{9}{|c|}{ Nectariniidae } \\
\hline 280. & Purple-rumped Sunbird & Nectarinia zeylonica (Linnaeus) & $\mathrm{R}$ & $\sqrt{ }$ & $\sqrt{ }$ & $\sqrt{ }$ & $\sqrt{ }$ & $\sqrt{ }$ \\
\hline 281. & Small Sunbird & Nectarinia minima (Sykes) & $\mathrm{R}$ & $\sqrt{ }$ & & & $\sqrt{ }$ & $\sqrt{ }$ \\
\hline 282. & Loten's Sunbird & Nectarinia lotenia (Linnaeus) & $\mathrm{R}$ & $\sqrt{ }$ & $\sqrt{ }$ & $\sqrt{ }$ & $\sqrt{ }$ & $\sqrt{ }$ \\
\hline 283. & Purple Sunbird & Nectarinia asiatica Latham & $\mathrm{R}$ & $\sqrt{ }$ & $\sqrt{ }$ & $\sqrt{ }$ & $\sqrt{ }$ & $\sqrt{ }$ \\
\hline 284. & Little Spiderhunter & Arachnothera longirostra (Latham) & $\mathrm{R}$ & & & & $\sqrt{ }$ & $\sqrt{ }$ \\
\hline \multicolumn{9}{|c|}{ Estrildidae } \\
\hline 285. & Red Munia & Amandava amandava (Linnaeus) & $\mathrm{R}$ & $\sqrt{ }$ & & & & $\sqrt{ }$ \\
\hline 286. & White-throated Munia & Lonchura malabarica (Linnaeus) & $\mathrm{R}$ & $\sqrt{ }$ & & & & $\sqrt{ }$ \\
\hline 287. & White-rumped Munia & Lonchura striata (Linnaeus) & $\mathrm{R}$ & $\sqrt{ }$ & $\sqrt{ }$ & $\sqrt{ }$ & $\sqrt{ }$ & $\sqrt{ }$ \\
\hline 288. & Black-throated Munia & Lonchura kelaarti (Jerdon) & $\mathrm{R}$ & $\sqrt{ }$ & & & & $\sqrt{ }$ \\
\hline 289. & Black-headed Munia & Lonchura malacca (Linnaeus) & $\mathrm{R}$ & $\sqrt{ }$ & & & & \\
\hline 290. & Spotted Munia & Lonchura punctulata (Linnaeus) & $\mathrm{R}$ & $\sqrt{ }$ & & $\sqrt{ }$ & & $\sqrt{ }$ \\
\hline \multicolumn{9}{|c|}{ Passerinae } \\
\hline 291. & Yellow-throated Sparrow & Petronia xanthocollis (Burton) & $\mathrm{R}$ & & $\sqrt{ }$ & $\sqrt{ }$ & & \\
\hline 292. & House Sparrow & Passer domesticus (Linnaeus) & $\mathrm{R}$ & & & & & $\sqrt{ }$ \\
\hline \multicolumn{9}{|c|}{ Ploceinae } \\
\hline 293. & Baya Weaver & Ploceus philippinus (Linnaeus) & $\mathrm{R}$ & $\sqrt{ }$ & $\sqrt{ }$ & $\sqrt{ }$ & & \\
\hline 294. & Streaked Weaver & Ploceus manyar (Horsfield) & $\mathrm{R}$ & $\sqrt{ }$ & $\sqrt{ }$ & & & \\
\hline \multicolumn{9}{|c|}{ Sturnidae } \\
\hline 295. & Grey-headed Starling & Sturnus malabaricus (Gmelin) & $\mathrm{R}$ & $\sqrt{ }$ & & $\sqrt{ }$ & $\sqrt{ }$ & $\sqrt{ }$ \\
\hline 296. & Brahminy Starling & Sturnus pagodarum Gmelin) & $\mathrm{R}$ & & & $\sqrt{ }$ & & $\sqrt{ }$ \\
\hline 297. & Rosy Starling & Sturnus roseus (Linnaeus) & M & & & $\sqrt{ }$ & & $\sqrt{ }$ \\
\hline 298. & Common Myna & Acridotheres tristis (Linnaeus) & $\mathrm{R}$ & $\sqrt{ }$ & $\sqrt{ }$ & $\sqrt{ }$ & $\sqrt{ }$ & $\sqrt{ }$ \\
\hline 299. & Jungle Myna & Acridotheres fuscus (Wagler) & $\mathrm{R}$ & $\sqrt{ }$ & $\sqrt{ }$ & $\sqrt{ }$ & $\sqrt{ }$ & $\sqrt{ }$ \\
\hline 300. & Common Hill Myna & Gracula religiosa Linnaeus & $\mathrm{R}$ & & & & $\sqrt{ }$ & $\sqrt{ }$ \\
\hline \multicolumn{9}{|c|}{ Oriolidae } \\
\hline 301. & Eurasian Golden Oriole & Oriolus oriolus (Linnaeus) & LM & $\sqrt{ }$ & $\sqrt{ }$ & $\sqrt{ }$ & $\sqrt{ }$ & $\sqrt{ }$ \\
\hline 302. & Black-naped Oriole & Oriolus chinensis (Linnaeus) & $\mathrm{R}$ & & & & $\sqrt{ }$ & $\sqrt{ }$ \\
\hline 303. & Black-headed Oriole & Oriolus xanthornus (Linnaeus) & $\mathrm{R}$ & $\sqrt{ }$ & $\sqrt{ }$ & $\sqrt{ }$ & $\sqrt{ }$ & $\sqrt{ }$ \\
\hline \multicolumn{9}{|c|}{ Dicruridae } \\
\hline 304. & Black Drongo & Dicrurus macrocercus Vieillot & $\mathrm{R}$ & $\sqrt{ }$ & $\sqrt{ }$ & $\sqrt{ }$ & $\sqrt{ }$ & $\sqrt{ }$ \\
\hline 305. & Ashy Drongo & Dicrurus leucophaeus Vieillot & $\mathrm{R}$ & $\sqrt{ }$ & $\sqrt{ }$ & $\sqrt{ }$ & $\sqrt{ }$ & $\sqrt{ }$ \\
\hline 306. & Whitebellied Drongo & Dicrurus caerulescens (Linnaeus) & $\mathrm{R}$ & $\sqrt{ }$ & $\sqrt{ }$ & $\sqrt{ }$ & $\sqrt{ }$ & $\sqrt{ }$ \\
\hline 307. & Bronzed Drongo & Dicrurus aeneus Vieillot & $\mathrm{R}$ & & & $\sqrt{ }$ & $\sqrt{ }$ & $\sqrt{ }$ \\
\hline 308. & Spangled Drongo & Dicrurus hottentottus (Linnaeus) & $\mathrm{R}$ & & & & $\sqrt{ }$ & $\sqrt{ }$ \\
\hline 309. & Greater Racket-tailed Drongo & Dicrurus paradiseus (Linnaeus) & $\mathrm{R}$ & & $\sqrt{ }$ & $\sqrt{ }$ & $\sqrt{ }$ & $\sqrt{ }$ \\
\hline \multicolumn{9}{|c|}{ Artamidae } \\
\hline 310. & Ashy Woodswallow & Artamus fuscus Vieillot & $\mathrm{R}$ & $\sqrt{ }$ & $\sqrt{ }$ & $\sqrt{ }$ & $\sqrt{ }$ & $\sqrt{ }$ \\
\hline \multicolumn{9}{|c|}{ Corvidae } \\
\hline 311. & Indian Tree Pie & Dendrocitta vagabunda (Latham) & $\mathrm{R}$ & $\sqrt{ }$ & $\sqrt{ }$ & $\sqrt{ }$ & $\sqrt{ }$ & $\sqrt{ }$ \\
\hline 312. & House Crow & Corvus splendens Vieillot & $\mathrm{R}$ & $\sqrt{ }$ & $\sqrt{ }$ & $\sqrt{ }$ & $\sqrt{ }$ & $\sqrt{ }$ \\
\hline 313. & Jungle Crow & Corvus macrorhynchos Wagler & $\mathrm{R}$ & $\sqrt{ }$ & $\sqrt{ }$ & $\sqrt{ }$ & $\sqrt{ }$ & $\sqrt{ }$ \\
\hline
\end{tabular}

R - Resident; M - Migrant; LM - Local Migrant; S - Straggler; S1 - Kole wetlands; S2 - KFRI Campus; S3 - KAU campus; S4 - Chimmoni Wildlife Sanctuary; S5 - Peechi-Vazhani Wildlife Sanctuary; Ö - Present 
Table 3. Occurrence of bird species in Thrissur district, Kerala

\begin{tabular}{|c|c|c|c|c|}
\hline Order & World ${ }^{1}$ & $\begin{array}{l}\text { Indian sub } \\
\text { continent }{ }^{2}\end{array}$ & $\begin{array}{l}\text { Kerala } \\
\text { State }^{3}\end{array}$ & $\begin{array}{l}\text { Thrissur } \\
\text { District }{ }^{4}\end{array}$ \\
\hline Archaeopterygiformes* & 01 & - & - & - \\
\hline Hesperornithiformes ${ }^{* *}$ & 07 & - & - & - \\
\hline Ichthyornithiformes ${ }^{* *}$ & 09 & - & - & - \\
\hline Struthioniformes & 01 & - & - & - \\
\hline Rheiformes & 02 & - & - & - \\
\hline Casuariiformes & 04 & - & - & - \\
\hline Aepyornithiformes** & 04 & - & - & - \\
\hline Dinornithiformes** & 12 & - & - & - \\
\hline Apterygiformes & 08 & - & - & - \\
\hline Tinamiformes & 50 & - & - & - \\
\hline Sphenisciformes & 18 & - & - & - \\
\hline Gaviiformes & 05 & 02 & - & - \\
\hline Podicipediformes & 22 & 05 & 02 & 01 \\
\hline Procellariiformes & 32 & 21 & 02 & - \\
\hline Odontopterygiformes ${ }^{* *}$ & 03 & - & - & - \\
\hline Pelecaniformes & 45 & 17 & 10 & 07 \\
\hline Ciconiiformes & 88 & 34 & 26 & 22 \\
\hline Phoenicopteriformes & 06 & 02 & - & - \\
\hline Anseriformes & 145 & 44 & 12 & 10 \\
\hline Falconiformes & 285 & 71 & 40 & 19 \\
\hline Galliformes & 235 & 49 & 12 & 05 \\
\hline Diatrymiformes & 04 & - & - & - \\
\hline Gruiformes & 186 & 33 & 14 & 07 \\
\hline Charadriiformes & 199 & 121 & 70 & 58 \\
\hline Columbiformes & 274 & 40 & 13 & 10 \\
\hline Psittaciformes & 330 & 15 & 05 & 04 \\
\hline Cuculiformes & 133 & 24 & 15 & 09 \\
\hline Strigiformes & 129 & 33 & 15 & 09 \\
\hline Caprimulgiformes & 96 & 11 & 06 & 05 \\
\hline Apodiformes & 403 & 17 & 08 & 07 \\
\hline Coliiformes & 06 & - & - & - \\
\hline Trogoniformes & 40 & 03 & 01 & 01 \\
\hline Coraciiformes & 183 & 33 & 19 & 14 \\
\hline Piciformes & 343 & 46 & 18 & 13 \\
\hline Passeriformes & 4978 & 719 & 187 & 112 \\
\hline Total & 8286 & 1340 & 475 & 313 \\
\hline
\end{tabular}

Source: 1 - Harrison (1978); 2 - Ali \& Ripley (1983), Manakadan \& Pittie (2001) 3 - Ali, 1969, Neelakantan et al. 1993; 4 - Present study

${ }^{*}$ Fossil bird; ** Extinct birds

Platalea leucorodia Linn. in Kerala. Journal of the Bombay Natural History Society 87(2): 289.

Jayson, E.A. (2000). Occurrence of Black Stork Ciconia nigra (Linnaeus) in Kole wetlands of Thrissur, Kerala. Newsletter for Birdwatchers 40(3): 39-40.

Jayson, E.A. and P.S. Easa (1996). Status, food and feeding habits of larger mammals in Chimmony Wildlife Sanctuary. KFRI Research Report No.108. Kerala Forest Research Institute, 49pp.

Jayson, E.A. and D.N. Mathew (2000a). Seasonal changes of tropical forest birds in southern Western Ghats. Journal of the Bombay Natural History Society 97: 52-61.

Jayson, E.A. and D.N. Mathew (2000b). Diversity and species abundance distribution of birds in the tropical forests of Silent Valley, Kerala. Journal of the Bombay Natural History Society 97: 52-61.

Jayson, E.A. and D.N. Mathew (2002). Structure and composition of two bird communities in the Southern Western Ghats. Journal of the Bombay Natural History Society 99(1): 8-25.

Jayson, E.A. and D.N. Mathew (2003). Vertical stratification and its relation to foliage in tropical forest birds in Western Ghats (India). Acta Ornithologica 38: 111-116.

Jayson, E.A. and C. Sivaperuman (2003). Sighting of Lesser Frigate Bird Fregata ariel Gray in the Kole Wetlands of Thrissur, Kerala. Journal of the Bombay Natural History Society 100(1): 106-108.

Jayson, E.A., K.K. Ramachandran and C. Sivaperuman (2000). Avifauna of KFRI campus. Evergreen 44: 19-20.

Joseph, K.J. 1999. Forest Wagtail, Yellow Wagtail and Indian Whiskered
Tern. Newsletter for Birdwatchers 39(3): 50.

Manakadan, R. and A. Pittie (2001). Standardised common and scientific names of the Birds of the Indian Subcontinent. Buceros 6(1): $1-37$.

Nameer, P.O., R. Resmi Nair, K.R. Anoop, S.G. Nair, R. Lekshmi and P. Radhakrishnan (2000). Birds of Kerala Agricultural University Campus, Thrissur. Zoos' Print Journal 15(4): 243-246.

Neelakantan, K.K., C. Sasikumar and R. Venugopalan (1993). $A$ Book of Kerala Birds. World Wide Fund for Nature-India, Kerala State Committee, Trivandrum, 146pp.

Perennou, C. (1990). Species to look for in India. Newsletter for Birdwatchers 30(5\&6): 10 .

Ravindran, P.K. (2001). Occurrence of the White-winged Black Tern Chilidonias leucopterus in Kerala. Journal of the Bombay Natural History Society 98(1): 112-113.

Santharam, V. (1995a). Ecology of sympatric Woodpecker species of Western Ghats, India. Ph.D. Dissertation, Pondicherry University, Pondicherry, India, 162pp.

Santharam, V. (1995b). The Greythroated or Plain Sand Marten Riparia paludicola Vieillot - a new bird for Southern India. Journal of the Bombay Natural History Society 92(1): 122.

Sasidharan, N. and V.V. Sivarajan (1996). Flowering Plants of Thrissur Forests (Western Ghats, Kerala, India). Scientific Publishers, Jodhpur, 579pp.

Satheesan, S.M. (1990). The ecology and behaviour of the Pariah Kite (Milvus migrans govinda) Sykes as a problem bird at some Indian aerodromes. Ph.D. Dissertation, University of Bombay, Bombay, India, 248 pp.

Shukkur, E.A.A. and K.J. Joseph (1980). Breeding biology of the Black Drongo. Journal of the Bombay Natural History Society 75(Suppl.): $1212-1226$.

Sivaperuman, C. and E.A. Jayson (2000). Birds of Kole Wetlands, Thrissur, Kerala. Zoos' Print Journal 15(10): 344-349.

Sivaperuman, C. and E.A. Jayson (2001). Structure and species composition of wetland birds in the Kole lands of Thrissur, Kerala. Proceedings of the Thirteenth Kerala Science Congress, 2001, Thrissur. pp. $152-155$.

Sivaperuman, C. and E.A. Jayson (2002). Occurrence of Northern Shoveller Anas clypeata Linnaeus in Kole Wetlands of Thrissur, Kerala. Journal of Bombay natural History Society 99(3): 517.

Vijayan, L. (1984). Comparative biology of Drongos (Family: Dicruridae, Class: Aves) with special reference to ecological isolation. Ph.D. Dissertation, University of Bombay, Bombay, India, 405pp.

Vijayan, V.S. (1979). Parambikulam Wildlife Sanctuary and its adjacent area. Journal of the Bombay Natural History Society 75: 888-900.

Yahya, H.S.A. (1980). A comparative study of ecology and biology of barbets, Megalaima spp. (Capitonidae: Piciformes) with special reference to (Megalaima viridis) (Boddaert) and (M. rubricapilla malabarica) (Blyth) at Periyar Tiger Reserve, Kerala. Ph.D. Dissertation, University of Bombay, Bombay, 210pp.

Zacharias, V.J. (1979). Ecology and biology of certain species of Indian Babblers (Turdoides spp.) in Malabar. Ph.D. Dissertation, University of Calicut, Kerala, 196p.

Zacharias, V.J. and D.N. Mathew (1988). Ecology of Babblers (Turdoides spp.). Journal of the Bombay Natural History Society 85(1): 50-63.

\section{ACKnowledgements}

We are grateful to Dr. J.K. Sharma, Director, Kerala Forest Research Institute, Peechi, Kerala for valuable advice and suggestions. The authors are thankful to the Kerala Forest and Wildlife Department for sponsoring the studies at Chimmony Wildlife Sanctuary and Kole wetlands of Thrissur. Dr. C. Renuka, Programme Coordinator, Division of Forest Ecology and Biodiversity Conservation is acknowledged for the encouragement and Dr. V.V. Sudheendrakumar for suggesting improvements in an earlier draft. 